\title{
Combinatorial Expressions for the Tau Functions of $q$-Painlevé V and III Equations
}

\author{
Yuya MATSUHIRA and Hajime NAGOYA
}

School of Mathematics and Physics, Kanazawa University, Kanazawa, Ishikawa 920-1192, Japan

E-mail: y.matsu0727@gmail.com,nagoya@se.kanazawa-u.ac.jp

Received November 24, 2018, in final form September 13, 2019; Published online September 23, 2019

https://doi.org/10.3842/SIGMA.2019.074

\begin{abstract}
We derive series representations for the tau functions of the $q$-Painlevé $\mathrm{V}, \mathrm{III}_{1}$, $\mathrm{III}_{2}$, and $\mathrm{III}_{3}$ equations, as degenerations of the tau functions of the $q$-Painlevé VI equation in [Jimbo M., Nagoya H., Sakai H., J. Integrable Syst. 2 (2017), xyx009, 27 pages]. Our tau functions are expressed in terms of $q$-Nekrasov functions. Thus, our series representations for the tau functions have explicit combinatorial structures. We show that general solutions to the $q$-Painlevé $\mathrm{V}, \mathrm{III}_{1}, \mathrm{III}_{2}$, and $\mathrm{III}_{3}$ equations are written by our tau functions. We also prove that our tau functions for the $q$-Painlevé $\mathrm{III}_{1}, \mathrm{III}_{2}$, and $\mathrm{III}_{3}$ equations satisfy the three-term bilinear equations for them.
\end{abstract}

Key words: q-Painlevé equations; tau functions; $q$-Nekrasov functions; bilinear equations

2010 Mathematics Subject Classification: 39A13; 33E17; 05A30

\section{Introduction}

The $q$-Painlevé equations $[17,24]$ are $q$-difference analogs of the Painlevé equations, which were introduced as new special functions beyond elliptic functions and the hypergeometric functions more than one hundred years ago [12, 22, 23], and are now considered as important special functions with many applications both in mathematics and physics.

Similarly, as for other integrable systems, tau functions play a crucial role in the studies of the Painelvé equations. The recent discovery by [10] states that the tau function of the sixth Painlevé equation is a Fourier transform of Virasoro conformal blocks with $c=1$, which admit explicit combinatorial formulas by AGT correspondence [1]. Series representations of the tau functions of other types are also studied in $[8,11,20,21]$ for differential cases, $[5,6,15]$ for $q$-difference cases.

In [15], a general solution $(y, z)$ to the $q$-Painlevé VI equation [16] was expressed by the tau functions having $q$-Nekrasov type expressions, and it was conjectured that the tau functions satisfy the bilinear equations for the $q$-Painlevé VI equation. In this paper, we give explicit expressions for general solutions to the $q$-Painlevé $\mathrm{V}, \mathrm{III}_{1}, \mathrm{III}_{2}$, and $\mathrm{III}_{3}$ equations using degenerations of the tau functions of the $q$-Painlevé VI equation. We also give conjectures on the bilinear equations satisfied by the tau functions of the $q$-Painlevé $\mathrm{V}$ equation and prove that the tau functions of the $q$-Painlevé $\mathrm{III}_{1}, \mathrm{III}_{2}$, and $\mathrm{III}_{3}$ equations satisfy the bilinear equations.

Our $q$-difference equations are as follows.

(i) the $q$-Painlevé VI equation:

$$
\frac{y \bar{y}}{a_{3} a_{4}}=\frac{\left(\bar{z}-b_{1} t\right)\left(\bar{z}-b_{2} t\right)}{\left(\bar{z}-b_{3}\right)\left(\bar{z}-b_{4}\right)}, \quad \frac{z \bar{z}}{b_{3} b_{4}}=\frac{\left(y-a_{1} t\right)\left(y-a_{2} t\right)}{\left(y-a_{3}\right)\left(y-a_{4}\right)} .
$$

(ii) the $q$-Painlevé $\mathrm{V}$ equation:

$$
\frac{y \bar{y}}{a_{3} a_{4}}=-\frac{\left(\bar{z}-b_{1} t\right)\left(\bar{z}-b_{2} t\right)}{\bar{z}-b_{3}}, \quad \frac{z \bar{z}}{b_{3}}=-\frac{\left(y-a_{1} t\right)\left(y-a_{2} t\right)}{a_{4}\left(y-a_{3}\right)} .
$$


(iii) the $q$-Painlevé III $_{1}$ equation:

$$
\frac{y \bar{y}}{a_{3} a_{4}}=-\frac{\bar{z}\left(\bar{z}-b_{2} t\right)}{\bar{z}-b_{3}}, \quad \frac{z \bar{z}}{b_{3}}=-\frac{y\left(y-a_{1} t\right)}{a_{4}\left(y-a_{3}\right)} .
$$

(iv) the $q$-Painlevé $\mathrm{III}_{2}$ equation:

$$
\frac{y \bar{y}}{a_{3} a_{4}}=-\frac{\bar{z}^{2}}{\bar{z}-b_{3}}, \quad \frac{z \bar{z}}{b_{3}}=-\frac{y\left(y-a_{2} t\right)}{a_{4}\left(y-a_{3}\right)} .
$$

(v-1) the $q$-Painlevé $\mathrm{III}_{3}$ equation of surface type $A_{7}^{(1) \prime}$ :

$$
\frac{y \bar{y}}{a_{3}}=\bar{z}^{2}, \quad z \bar{z}=-\frac{y\left(y-a_{2} t\right)}{y-a_{3}} .
$$

(v-2) the $q$-Painlevé $\mathrm{III}_{3}$ equation of surface type $A_{7}^{(1)}$ :

$$
\frac{y \bar{y}}{a_{3}}=-\frac{\bar{z}^{2}}{\bar{z}-b_{3}}, \quad z \bar{z}=\frac{y\left(y-a_{2} t\right)}{a_{2}} .
$$

Here, $y, z$ are functions of $t, \bar{y}=y(q t), \bar{z}=z(q t)$, and $a_{i}, b_{i}(i=1,2,3,4)$ are parameters.

From the point of view of Sakai's classification for the discrete Painlevé equations [25], the $q$-Painlevé VI, V, $\mathrm{III}_{1}, \mathrm{III}_{2}$ and $\mathrm{III}_{3}$ equations are derived from the symmetries/surfaces of type $D_{5}^{(1)} / A_{3}^{(1)}, A_{4}^{(1)} / A_{4}^{(1)}, E_{2}^{(1)} / A_{5}^{(1)}, E_{2}^{(1)} / A_{6}^{(1)}$ and $A_{1}^{(1)} / A_{7}^{(1)}$, respectively.

The degeneration scheme of Painlevé equations is as follows

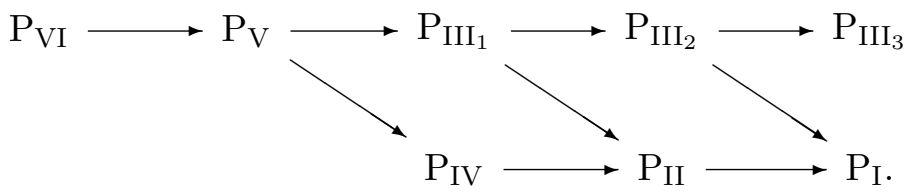

The degeneration pattern of the $q$-Painlevé equations we use is similar to the one in [19] but not exactly the same. Rather, our limiting procedure is a $q$-version for the one used in [11] in order to derive combinatorial expressions of tau functions of $\mathrm{P}_{\mathrm{V}}, \mathrm{P}_{\mathrm{III}_{1}}, \mathrm{P}_{\mathrm{III}_{2}}$, and $\mathrm{P}_{\mathrm{III}_{3}}$ from the Nekrasov type expression of the tau function of $\mathrm{P}_{\mathrm{VI}}[10]$.

For the case of the $q$-Painlevé $\mathrm{III}_{3}$ equation of surface type $A_{7}^{(1) \prime}$, a series representation for the tau function was proposed in [5], which are expressed by $q$-Virasoro Whittaker conformal blocks which equal Nekrasov partition functions for pure SU(2) 5d theory [3, 28]. A Fredholm determinant representation of the tau function by topological strings/spectral theory duality is proposed in [7]. For the $q$-Painlevé $\mathrm{III}_{3}$ equation of surface type $A_{7}^{(1)}$, a series representation for the tau function was proposed in [4]. Our tau functions for the $q$-Painlevé $\mathrm{III}_{3}$ equations obtained by the degeneration are equivalent to them.

Our plan is as follows. In Section 2, we recall the result on $q$-Painlevé VI equation in [15]. In Sections 3-6, we compute limits of tau functions and derive combinatorial expressions of general solutions and bilinear equations for $q$-Painlevé $\mathrm{V}, \mathrm{III}_{1}, \mathrm{III}_{2}$ and $\mathrm{III}_{3}$ equations.

Notations. Throughout the paper we fix $q \in \mathbb{C}^{\times}$such that $|q|<1$. We set

$$
\begin{aligned}
& {[u]=\left(1-q^{u}\right) /(1-q), \quad(a ; q)_{N}=\prod_{j=0}^{N-1}\left(1-a q^{j}\right),} \\
& \left(a_{1}, \ldots, a_{k} ; q\right)_{\infty}=\prod_{j=1}^{k}\left(a_{j} ; q\right)_{\infty}, \quad(a ; q, q)_{\infty}=\prod_{j, k=0}^{\infty}\left(1-a q^{j+k}\right) .
\end{aligned}
$$


We use the $q$-Gamma function and $q$-Barnes function defined by

$$
\Gamma_{q}(u)=\frac{(q ; q)_{\infty}}{\left(q^{u} ; q\right)_{\infty}}(1-q)^{1-u}, \quad G_{q}(u)=\frac{\left(q^{u} ; q, q\right)_{\infty}}{(q ; q, q)_{\infty}}(q ; q)_{\infty}^{u-1}(1-q)^{-(u-1)(u-2) / 2},
$$

which satisfy $\Gamma_{q}(1)=G_{q}(1)=1$ and

$$
\Gamma_{q}(u+1)=[u] \Gamma_{q}(u), \quad G_{q}(u+1)=\Gamma_{q}(u) G_{q}(u) .
$$

A partition is a finite sequence of positive integers $\lambda=\left(\lambda_{1}, \ldots, \lambda_{l}\right)$ such that $\lambda_{1} \geq \cdots \geq \lambda_{l}>0$. Denote the length of the partition by $\ell(\lambda)=l$. The conjugate partition $\lambda^{\prime}=\left(\lambda_{1}^{\prime}, \ldots, \lambda_{l^{\prime}}^{\prime}\right)$ is defined by $\lambda_{j}^{\prime}=\sharp\left\{i \mid \lambda_{i} \geq j\right\}, l^{\prime}=\lambda_{1}$. We regard a partition as a Young diagram. Namely, we regard a partition $\lambda$ also as the subset $\left\{(i, j) \in \mathbb{Z}^{2} \mid 1 \leq j \leq \lambda_{i}, i \geq 1\right\}$ of $\mathbb{Z}^{2}$, and denote its cardinality by $|\lambda|$. We denote the set of all partitions by $\mathbb{Y}$. For $\square=(i, j) \in \mathbb{Z}_{>0}^{2}$ we set $a_{\lambda}(\square)=\lambda_{i}-j$ (the arm length of $\square$ ) and $\ell_{\lambda}(\square)=\lambda_{j}^{\prime}-i$ (the leg length of $\square$ ). In the last formulas we set $\lambda_{i}=0$ if $i>\ell(\lambda)$ (resp. $\lambda_{j}^{\prime}=0$ if $j>\ell\left(\lambda^{\prime}\right)$ ). For a pair of partitions $(\lambda, \mu)$ and $u \in \mathbb{C}$ we set

$$
N_{\lambda, \mu}(u)=\prod_{\square \in \lambda}\left(1-q^{-\ell_{\lambda}(\square)-a_{\mu}(\square)-1} u\right) \prod_{\square \in \mu}\left(1-q^{\ell_{\mu}(\square)+a_{\lambda}(\square)+1} u\right),
$$

which we call a Nekrasov factor.

\section{Results on $q$-P $\mathrm{PI}_{\mathrm{VI}}$ from [15]}

In this section, we recall the results of [15] on the $q$-Painlevé VI equation. Define the tau function by

$$
\tau^{\mathrm{VI}}\left[\begin{array}{cc}
\theta_{1} & \theta_{t} \\
\theta_{\infty} & \theta_{0}
\end{array} \mid s, \sigma, t\right]=\sum_{n \in \mathbb{Z}} s^{n} t^{(\sigma+n)^{2}-\theta_{t}^{2}-\theta_{0}^{2}} C\left[\begin{array}{cc}
\theta_{1} & \theta_{t} \\
\theta_{\infty} & \theta_{0}
\end{array} \mid \sigma+n\right] Z\left[\begin{array}{cc}
\theta_{1} & \theta_{t} \\
\theta_{\infty} & \theta_{0}
\end{array} \mid \sigma+n, t\right]
$$

with the definition

$$
\begin{aligned}
& C\left[\begin{array}{cc}
\theta_{1} & \theta_{t} \\
\theta_{\infty} & \theta_{0}
\end{array} \mid \sigma\right]=\frac{\prod_{\varepsilon, \varepsilon^{\prime}= \pm} G_{q}\left(1+\varepsilon \theta_{\infty}-\theta_{1}+\varepsilon^{\prime} \sigma\right) G_{q}\left(1+\varepsilon \sigma-\theta_{t}+\varepsilon^{\prime} \theta_{0}\right)}{G_{q}(1+2 \sigma) G_{q}(1-2 \sigma)}, \\
& Z\left[\begin{array}{cc}
\theta_{1} & \theta_{t} \\
\theta_{\infty} & \theta_{0}
\end{array} \mid \sigma, t\right]=\sum_{\boldsymbol{\lambda}=\left(\lambda_{+}, \lambda_{-}\right) \in \mathbb{Y}^{2}} t^{|\boldsymbol{\lambda}|} . \frac{\prod_{\varepsilon, \varepsilon^{\prime}= \pm} N_{\varnothing, \lambda_{\varepsilon^{\prime}}}\left(q^{\varepsilon \theta_{\infty}-\theta_{1}-\varepsilon^{\prime} \sigma}\right) N_{\lambda_{\varepsilon}, \varnothing}\left(q^{\varepsilon \sigma-\theta_{t}-\varepsilon^{\prime} \theta_{0}}\right)}{\prod_{\varepsilon, \varepsilon^{\prime}= \pm} N_{\lambda_{\varepsilon}, \lambda_{\varepsilon^{\prime}}}\left(q^{\left(\varepsilon-\varepsilon^{\prime}\right) \sigma}\right)} .
\end{aligned}
$$

Put

$$
\begin{array}{rlrl}
\tau_{1}^{\mathrm{VI}} & =\tau^{\mathrm{VI}}\left[\begin{array}{ccc}
\theta_{1} & \theta_{t} \\
\theta_{\infty}+\frac{1}{2} & \theta_{0}
\end{array} \mid s, \sigma, t\right], & & \tau_{2}^{\mathrm{VI}}=\tau^{\mathrm{VI}}\left[\begin{array}{cc}
\theta_{1} & \theta_{t} \\
\theta_{\infty}-\frac{1}{2} & \theta_{0}
\end{array} \mid s, \sigma, t\right], \\
\tau_{3}^{\mathrm{VI}}=\tau^{\mathrm{VI}}\left[\begin{array}{cc}
\theta_{1} & \theta_{t} \\
\theta_{\infty} & \theta_{0}+\frac{1}{2}
\end{array} \mid s, \sigma+\frac{1}{2}, t\right], & \tau_{4}^{\mathrm{VI}}=\tau^{\mathrm{VI}}\left[\begin{array}{cc}
\theta_{1} & \theta_{t} \\
\theta_{\infty} & \theta_{0}-\frac{1}{2}
\end{array} \mid s, \sigma-\frac{1}{2}, t\right], \\
\tau_{5}^{\mathrm{VI}}=\tau^{\mathrm{VI}}\left[\begin{array}{cc}
\theta_{1}-\frac{1}{2} & \theta_{t} \\
\theta_{\infty} & \theta_{0}
\end{array} \mid s, \sigma, t\right], & \tau_{6}^{\mathrm{VI}}=\tau^{\mathrm{VI}}\left[\begin{array}{cc}
\theta_{1}+\frac{1}{2} & \theta_{t} \\
\theta_{\infty} & \theta_{0}
\end{array} \mid s, \sigma, t\right], \\
\tau_{7}^{\mathrm{VI}}=\tau^{\mathrm{VI}}\left[\begin{array}{cc}
\theta_{1} & \theta_{t}-\frac{1}{2} \\
\theta_{\infty} & \theta_{0}
\end{array} \mid s, \sigma+\frac{1}{2}, t\right], & \tau_{8}^{\mathrm{VI}}=\tau^{\mathrm{VI}}\left[\begin{array}{cc}
\theta_{1} & \theta_{t}+\frac{1}{2} \\
\theta_{\infty} & \theta_{0}
\end{array} \mid s, \sigma-\frac{1}{2}, t\right] .
\end{array}
$$

Here and after we write $\bar{f}(t)=f(q t), \underline{f}(t)=f(t / q)$. 
Theorem 2.1 ([15]). The functions $y$ and $z$ defined by

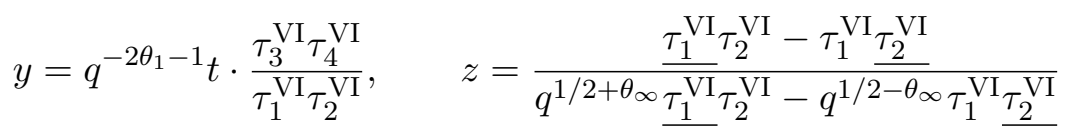

are solutions to the q-Painlevé VI equation

$$
\frac{y \bar{y}}{a_{3} a_{4}}=\frac{\left(\bar{z}-b_{1} t\right)\left(\bar{z}-b_{2} t\right)}{\left(\bar{z}-b_{3}\right)\left(\bar{z}-b_{4}\right)}, \quad \frac{z \bar{z}}{b_{3} b_{4}}=\frac{\left(y-a_{1} t\right)\left(y-a_{2} t\right)}{\left(y-a_{3}\right)\left(y-a_{4}\right)},
$$

with the parameters

$$
\begin{array}{lrr}
a_{1}=q^{-2 \theta_{1}-1}, & a_{2}=q^{-2 \theta_{t}-2 \theta_{1}-1}, & a_{3}=q^{-1}, \quad a_{4}=q^{-2 \theta_{1}-1}, \\
b_{1}=q^{-\theta_{0}-\theta_{t}-\theta_{1}}, & b_{2}=q^{\theta_{0}-\theta_{t}-\theta_{1}}, & b_{3}=q^{\theta_{\infty}-1 / 2},
\end{array}
$$

The formula for $y$ above can be regarded as an extension of Mano's asymptotic expansion to all orders for the solution of $q-\mathrm{P}_{\mathrm{VI}}$ [18]. Theorem 2.1 was obtained by constructing the fundamental solution of the Lax-pair for $q$ - $\mathrm{P}_{\mathrm{VI}}$ in [16], in terms of $q$-conformal blocks in [2]. The method of construction of the fundamental solution is a $q$-analogue of the CFT approach used in [14]. In the derivation of Theorem 2.1 convergence of the fundamental solution was assumed and it has not been proved. Recently, analyticity of K-theoretic Nekrasov functions in cases different from our case was discussed in [9]. We remark that the convergence of the pure $q$-Nekrasov partition function with $q_{1} q_{2}=1$ on $\mathbb{C}$ is proved in [5].

Conjecture $2.2([15])$. The tau functions $\tau_{i}^{\mathrm{VI}}(i=1, \ldots, 8)$ satisfy the following bilinear equations

$$
\begin{aligned}
& \tau_{1}^{\mathrm{VI}} \tau_{2}^{\mathrm{VI}}-q^{-2 \theta_{1}} t \tau_{3}^{\mathrm{VI}} \tau_{4}^{\mathrm{VI}}-\left(1-q^{-2 \theta_{1}} t\right) \tau_{5}^{\mathrm{VI}} \tau_{6}^{\mathrm{VI}}=0, \\
& \tau_{1}^{\mathrm{VI}} \tau_{2}^{\mathrm{VI}}-t \tau_{3}^{\mathrm{VI}} \tau_{4}^{\mathrm{VI}}-\left(1-q^{-2 \theta_{t}} t\right) \tau_{5}^{\mathrm{VI}} \overline{\tau_{6}^{\mathrm{VI}}}=0, \\
& \tau_{1}^{\mathrm{VI}} \tau_{2}^{\mathrm{VI}}-\tau_{3}^{\mathrm{VI}} \tau_{4}^{\mathrm{VI}}+\left(1-q^{-2 \theta_{1}} t\right) q^{2 \theta_{t}} \tau_{7}^{\mathrm{VI}} \overline{\tau_{8}^{\mathrm{VI}}}=0 \text {, } \\
& \tau_{1}^{\mathrm{VI}} \tau_{2}^{\mathrm{VI}}-q^{2 \theta_{t}} \tau_{3}^{\mathrm{VI}} \tau_{4}^{\mathrm{VI}}+\left(1-q^{-2 \theta_{t}} t\right) q^{2 \theta_{t}} \tau_{7}^{\mathrm{VI}} \tau_{8}^{\mathrm{VI}}=0 \text {, } \\
& \tau_{5}^{\mathrm{VI}} \tau_{6}^{\mathrm{VI}}+q^{-\theta_{1}-\theta_{\infty}+\theta_{t}-1 / 2} t \tau_{7}^{\mathrm{VI}} \tau_{8}^{\mathrm{VI}}-\tau_{1}^{\mathrm{VI}} \tau_{2}^{\mathrm{VI}}=0, \\
& \tau_{5}^{\mathrm{VI}} \tau_{6}^{\mathrm{VI}}+q^{-\theta_{1}+\theta_{\infty}+\theta_{t}-1 / 2} t \tau_{7}^{\mathrm{VI}} \tau_{8}^{\mathrm{VI}}-\tau_{1}^{\mathrm{VI}} \tau_{2}^{\mathrm{VI}}=0, \\
& \overline{\tau_{5}^{\mathrm{VI}}} \tau_{6}^{\mathrm{VI}}+q^{\theta_{0}+2 \theta_{t}} \tau_{7}^{\mathrm{VI}} \tau_{8}^{\mathrm{VI}}-q^{\theta_{t}} \tau_{3}^{\mathrm{VI}} \tau_{4}^{\mathrm{VI}}=0, \\
& \overline{\tau_{5}^{\mathrm{VI}}} \tau_{6}^{\mathrm{VI}}+q^{-\theta_{0}+2 \theta \theta_{t}} \tau_{7}^{\mathrm{VI}} \tau_{8}^{\mathrm{VI}}-q^{\theta_{t}} \tau_{3}^{\mathrm{VI}} \tau_{4}^{\mathrm{VI}}=0 .
\end{aligned}
$$

Then, the function $y, z$

$$
y=q^{-2 \theta_{1}-1} t \frac{\tau_{3}^{\mathrm{VI}} \tau_{4}^{\mathrm{VI}}}{\tau_{1}^{\mathrm{VI}} \tau_{2}^{\mathrm{VI}}}, \quad z=-q^{\theta_{t}-\theta_{1}-1} t \frac{\tau_{7}^{\mathrm{VI}} \tau_{8}^{\mathrm{VI}}}{\underline{\tau_{5}^{\mathrm{VI}}} \tau_{6}^{\mathrm{VI}}}
$$

solves $q-\mathrm{P}_{\mathrm{VI}}(2.2)$.

The function $y$ in Conjecture 2.2 is expressed in the same form in Theorem 2.1, while the function $z$ in Conjecture 2.2 is not. By the bilinear equations (2.7) and (2.8), we obtain the expression of $z$ in (2.11) from the expression of $z$ in (2.1).

We note that in [15] we have a Lax pair with respect to the shift $t \rightarrow q t$, namely, a fundamental solution of the linear $q$-difference equations

$$
Y(q x, t)=A(x, t) Y(x, t), \quad Y(x, q t)=B(x, t) Y(x, t)
$$

for certain 2 by 2 matrices $A(x, t)$ and $B(x, t)$ was constructed in terms of $q$-Nekrasov functions. From (2.12) we obtain the four-term bilinear equation in [15, Remark 3.5]:

$$
\underline{\tau_{1}^{\mathrm{VI}}} \tau_{2}^{\mathrm{VI}}-\tau_{1}^{\mathrm{VI}} \underline{\tau_{2}^{\mathrm{VI}}}=\frac{q^{1 / 2+\theta_{\infty}}-q^{1 / 2-\theta_{\infty}}}{q^{-\theta_{0}}-q^{\theta_{0}}} q^{-\theta_{1}-1} t\left(\underline{\tau_{3}^{\mathrm{VI}}} \tau_{4}^{\mathrm{VI}}-\tau_{3}^{\mathrm{VI}} \underline{\tau_{4}^{\mathrm{VI}}}\right) .
$$




\section{From $q-\mathrm{P}_{\mathrm{VI}}$ to $q-\mathrm{P}_{\mathrm{V}}$}

In this section, we take a limit of the tau functions of $q-\mathrm{P}_{\mathrm{VI}}$ to $q-\mathrm{P}_{\mathrm{V}}$. Define the tau function by

$$
\tau^{\mathrm{V}}\left(\theta_{*}, \theta_{t}, \theta_{0} \mid s, \sigma, t\right)=\sum_{n \in \mathbb{Z}} s^{n} t^{(\sigma+n)^{2}-\theta_{t}^{2}-\theta_{0}^{2}} C_{\mathrm{V}}\left[\theta_{*}, \theta_{t}, \theta_{0} \mid \sigma+n\right] Z_{\mathrm{V}}\left[\theta_{*}, \theta_{t}, \theta_{0} \mid \sigma+n, t\right],
$$

with

$$
\begin{aligned}
& C_{\mathrm{V}}\left[\theta_{*}, \theta_{t}, \theta_{0} \mid \sigma\right]=(q-1)^{-\sigma^{2}} \prod_{\varepsilon= \pm} \frac{G_{q}\left(1-\theta_{*}+\varepsilon \sigma\right)}{G_{q}(1+2 \varepsilon \sigma)} \prod_{\varepsilon, \varepsilon^{\prime}= \pm} G_{q}\left(1+\varepsilon \sigma-\theta_{t}+\varepsilon^{\prime} \theta_{0}\right), \\
& Z_{\mathrm{V}}\left[\theta_{*}, \theta_{t}, \theta_{0} \mid \sigma, t\right]=\sum_{\left(\lambda_{+}, \lambda_{-}\right) \in \mathbb{Y}^{2}} t^{\left|\lambda_{+}\right|+\left|\lambda_{-}\right|} \frac{\prod_{\varepsilon= \pm} N_{\varnothing, \lambda_{\varepsilon}}\left(q^{-\theta_{*}-\varepsilon \sigma}\right) f_{\lambda_{\varepsilon}}\left(q^{\varepsilon \sigma}\right) \prod_{\varepsilon, \varepsilon^{\prime}= \pm} N_{\lambda_{\varepsilon}, \varnothing}\left(q^{\varepsilon \sigma-\theta_{t}-\varepsilon^{\prime} \theta_{0}}\right)}{\prod_{\varepsilon, \varepsilon^{\prime}= \pm} N_{\lambda_{\varepsilon}, \lambda_{\varepsilon^{\prime}}}\left(q^{\left(\varepsilon-\varepsilon^{\prime}\right) \sigma}\right)},
\end{aligned}
$$

where

$$
f_{\lambda}(u)=\prod_{\square \in \lambda}\left(-q^{\ell_{\lambda}(\square)+a_{\varnothing}(\square)+1} u^{-1}\right) .
$$

We remark that the factor $f_{\lambda}(u)$ corresponds to the five-dimensional Chern-Simons term. The Chern-Simons term in [27] reads as

$$
\exp \left(-\beta \sum_{k} \sum_{(i, j) \in Y_{k}}\left(a_{k}+\epsilon(i-j)\right)\right)
$$

where $\beta, a_{k}$ are parameters and $Y_{1}, \ldots, Y_{N}$ are Young tableaux labelling the fixed points. See [27] for the details. Since

$$
\sum_{\square \in \lambda} \ell_{\lambda}(\square)+a_{\varnothing}(\square)+1=\sum_{(i, j) \in \lambda} \lambda_{j}^{\prime}-i-j+1=\sum_{(i, j) \in \lambda} i-j,
$$

they coincide when $N=2$. It is possible to remove $f_{\lambda_{\varepsilon}}\left(q^{\varepsilon \sigma}\right)$ from $Z_{\mathrm{V}}\left[\theta_{*}, \theta_{t}, \theta_{0} \mid \sigma, t\right]$ by change of variables. Because if we set

$$
Z_{\mathrm{V}}^{C S=0}\left[\theta_{*}, \theta_{t}, \theta_{0} \mid \sigma, t\right]=\sum_{\left(\lambda_{+}, \lambda_{-}\right) \in \mathbb{Y}^{2}} t^{\left|\lambda_{+}\right|+\left|\lambda_{-}\right|} \frac{\prod_{\varepsilon= \pm} N_{\varnothing, \lambda_{\varepsilon}}\left(q^{-\theta_{*}-\varepsilon \sigma}\right) \prod_{\varepsilon, \varepsilon^{\prime}= \pm} N_{\lambda_{\varepsilon}, \varnothing}\left(q^{\varepsilon \sigma-\theta_{t}-\varepsilon^{\prime} \theta_{0}}\right)}{\prod_{\varepsilon, \varepsilon^{\prime}= \pm} N_{\lambda_{\varepsilon}, \lambda_{\varepsilon^{\prime}}}\left(q^{\left(\varepsilon-\varepsilon^{\prime}\right) \sigma}\right)},
$$

then we have

$$
Z_{\mathrm{V}}\left[\theta_{*}, \theta_{t}, \theta_{0} \mid \sigma, t\right]=Z_{\mathrm{V}}^{C S=0}\left[-\theta_{*},-\theta_{t}, \theta_{0} \mid \sigma, q^{-\theta_{*}-2 \theta_{t}} t\right]
$$

from the relations $N_{\varnothing, \lambda}(u)=f_{\lambda}\left(u^{-1}\right) N_{\lambda, \varnothing}\left(u^{-1}\right), N_{\lambda, \varnothing}(u)=f_{\lambda}(u)^{-1} N_{\varnothing, \lambda}\left(u^{-1}\right)$, and $N_{\lambda, \mu}(u)=$ $N_{\mu^{\prime}, \lambda^{\prime}}(u)[15$, Lemma A.2].

We define tau functions for $q-\mathrm{P}_{\mathrm{V}}$ by

$$
\begin{array}{lll}
\tau_{1}^{\mathrm{V}}=\tau^{\mathrm{V}}\left(\theta_{*}-\frac{1}{2}, \theta_{t}, \theta_{0} \mid s, \sigma, t / \sqrt{q}\right), & \tau_{2}^{\mathrm{V}}=\tau^{\mathrm{V}}\left(\theta_{*}+\frac{1}{2}, \theta_{t}, \theta_{0} \mid s, \sigma, \sqrt{q} t\right), \\
\tau_{3}^{\mathrm{V}}=\tau^{\mathrm{V}}\left(\theta_{*}, \theta_{t}, \theta_{0}+\frac{1}{2} \mid s, \sigma+\frac{1}{2}, t\right), & \tau_{4}^{\mathrm{V}}=\tau^{\mathrm{V}}\left(\theta_{*}, \theta_{t}, \theta_{0}-\frac{1}{2} \mid s, \sigma-\frac{1}{2}, t\right), \\
\tau_{5}^{\mathrm{V}}=\tau^{\mathrm{V}}\left(\theta_{*}, \theta_{t}-\frac{1}{2}, \theta_{0} \mid s, \sigma+\frac{1}{2}, t\right), & \tau_{6}^{\mathrm{V}}=\tau^{\mathrm{V}}\left(\theta_{*}, \theta_{t}+\frac{1}{2}, \theta_{0} \mid s, \sigma-\frac{1}{2}, t\right) .
\end{array}
$$

Let

$$
C_{1}=C_{6}=(q-1)^{-\sigma^{2}} q^{-(\Lambda+1 / 2)\left(\sigma^{2}-\theta_{t}^{2}-\theta_{0}^{2}\right)} \prod_{\varepsilon= \pm} G_{q}\left(\frac{1}{2}-\Lambda+\varepsilon \sigma\right)^{-1}
$$




$$
\begin{aligned}
& C_{2}=C_{5}=(q-1)^{-\sigma^{2}} q^{-(\Lambda-1 / 2)\left(\sigma^{2}-\theta_{t}^{2}-\theta_{0}^{2}\right)} \prod_{\varepsilon= \pm} G_{q}\left(\frac{3}{2}-\Lambda+\varepsilon \sigma\right)^{-1}, \\
& C_{3}=(q-1)^{-(\sigma+1 / 2)^{2}} q^{-\Lambda\left((\sigma+1 / 2)^{2}-\theta_{t}^{2}-\left(\theta_{0}+1 / 2\right)^{2}\right)} \prod_{\varepsilon= \pm} G_{q}\left(1-\Lambda+\varepsilon\left(\sigma+\frac{1}{2}\right)\right)^{-1}, \\
& C_{4}=(q-1)^{-(\sigma-1 / 2)^{2}} q^{-\Lambda\left((\sigma-1 / 2)^{2}-\theta_{t}^{2}-\left(\theta_{0}-1 / 2\right)^{2}\right)} \prod_{\varepsilon= \pm} G_{q}\left(1-\Lambda+\varepsilon\left(\sigma-\frac{1}{2}\right)\right)^{-1}, \\
& C_{7}=(q-1)^{-(\sigma+1 / 2)^{2}} q^{-\Lambda\left((\sigma+1 / 2)^{2}-\left(\theta_{t}-1 / 2\right)^{2}-\theta_{0}^{2}\right)} \prod_{\varepsilon= \pm} G_{q}\left(1-\Lambda+\varepsilon\left(\sigma+\frac{1}{2}\right)\right)^{-1}, \\
& C_{8}=(q-1)^{-(\sigma-1 / 2)^{2}} q^{-\Lambda\left((\sigma-1 / 2)^{2}-\left(\theta_{t}+1 / 2\right)^{2}-\theta_{0}^{2}\right)} \prod_{\varepsilon= \pm} G_{q}\left(1-\Lambda+\varepsilon\left(\sigma-\frac{1}{2}\right)\right)^{-1} .
\end{aligned}
$$

Proposition 3.1. Set

$$
\begin{aligned}
& \theta_{1}+\theta_{\infty}=\Lambda, \quad \theta_{1}-\theta_{\infty}=\theta_{*}, \quad t=q^{\Lambda} t_{1}, \\
& s=\tilde{s}(q-1)^{-2 \sigma} q^{-2 \sigma \Lambda} \prod_{\varepsilon= \pm} \Gamma_{q}\left(\frac{1}{2}-\Lambda+\varepsilon \sigma\right)^{-\varepsilon} .
\end{aligned}
$$

Then we have

$$
\begin{aligned}
& C_{i} \tau_{i}^{\mathrm{VI}}\left(\theta_{\infty}, \theta_{1}, \theta_{t}, \theta_{0} \mid s, \sigma, t\right) \rightarrow \tau_{i}^{\mathrm{V}}\left(\theta_{*}, \theta_{t}, \theta_{0} \mid \tilde{s}, \sigma, t_{1}\right), \quad i=1,2,3,4, \\
& C_{5} \tau_{5}^{\mathrm{VI}}\left(\theta_{\infty}, \theta_{1}, \theta_{t}, \theta_{0} \mid s, \sigma, t\right) \rightarrow \tau_{1}^{\mathrm{V}}\left(\theta_{*}, \theta_{t}, \theta_{0} \mid \tilde{s}, \sigma, q t_{1}\right), \\
& C_{6} \tau_{6}^{\mathrm{VI}}\left(\theta_{\infty}, \theta_{1}, \theta_{t}, \theta_{0} \mid s, \sigma, t\right) \rightarrow \tau_{2}^{\mathrm{V}}\left(\theta_{*}, \theta_{t}, \theta_{0} \mid \tilde{s}, \sigma, t_{1} / q\right), \\
& C_{i} \tau_{i}^{\mathrm{VI}}\left(\theta_{\infty}, \theta_{1}, \theta_{t}, \theta_{0} \mid s, \sigma, t\right) \rightarrow \tau_{i-2}^{\mathrm{V}}\left(\theta_{*}, \theta_{t}, \theta_{0} \mid \tilde{s}, \sigma, t_{1}\right), \quad i=7,8,
\end{aligned}
$$

as $\Lambda \rightarrow \infty$. Here, we denote by $\tau_{i}^{\mathrm{VI}}\left(\theta_{\infty}, \theta_{1}, \theta_{t}, \theta_{0} \mid s, \sigma, t\right)$ the tau functions of $q$ - $\mathrm{P}_{\mathrm{VI}}$ presented in the previous section.

Proof. First, we verify the limit of the series part. For any partition $\lambda$ we have

$$
N_{\varnothing, \lambda}\left(q^{-\Lambda} u\right) q^{\Lambda|\lambda|}=\prod_{\square \in \lambda}\left(q^{\Lambda}-q^{\ell_{\lambda}(\square)+a_{\varnothing}(\square)+1} u\right) \rightarrow f_{\lambda}\left(u^{-1}\right), \quad \Lambda \rightarrow \infty .
$$

Hence, the series $Z\left[\begin{array}{cc}\theta_{1} & \theta_{t} \\ \theta_{\infty} & \theta_{0}\end{array} \mid \sigma, t\right]$ goes to $Z_{\mathrm{V}}\left[\theta_{*}, \theta_{t}, \theta_{0} \mid \sigma, t\right]$ as $\Lambda \rightarrow \infty$.

Second, we examine the limits of the coefficients of $Z$. By the identities (1.1) on $q$-Gamma function and $q$-Barnes function, for $n \in \mathbb{Z}$ we have

$$
\begin{aligned}
\prod_{\varepsilon= \pm} G_{q}(1-x+\varepsilon(\sigma+n))= & \prod_{\varepsilon= \pm} G_{q}(1-x+\varepsilon \sigma) \Gamma_{q}(-x+\varepsilon \sigma)^{\varepsilon n} \prod_{i=0}^{|n|-1}\left[-x+\frac{|n|}{n} \sigma\right] \\
& \times \prod_{i=0}^{|n|-1} \prod_{j=1}^{i}[-x+\sigma+j] \prod_{i=0}^{|n|-1} \prod_{j=1}^{i}[-x-\sigma-j] .
\end{aligned}
$$

Using the identity above, we compute the coefficient of $Z$ in $\tau_{1}^{\mathrm{VI}}$ multiplied by $C_{1}$ as follows

$$
\begin{aligned}
C_{1} s^{n} C & {\left[\begin{array}{cc}
\theta_{1} & \theta_{t} \\
\theta_{\infty}+\frac{1}{2} & \theta_{0}
\end{array} \mid \sigma+n\right] t^{(\sigma+n)^{2}-\theta_{t}^{2}-\theta_{0}^{2}} } \\
& =\tilde{s}^{n}(q-1)^{\sigma^{2}-2 \sigma n} q^{-\left(\sigma^{2}-\theta_{t}^{2}-\theta_{0}^{2}\right) / 2} t_{1}^{(\sigma+n)^{2}-\theta_{t}^{2}-\theta_{0}^{2}} q^{\Lambda n^{2}} \prod_{\varepsilon= \pm}\left(\frac{\Gamma_{q}\left(-\Lambda-\frac{1}{2}+\varepsilon \sigma\right)}{\Gamma_{q}\left(-\Lambda+\frac{1}{2}+\varepsilon \sigma\right)}\right)^{\varepsilon n}
\end{aligned}
$$




$$
\begin{aligned}
& \times \prod_{i=0}^{|n|-1}\left[-\Lambda-\frac{1}{2}+\frac{|n|}{n} \sigma\right] \prod_{i=0}^{|n|-1} \prod_{j=1}^{i}\left[-\Lambda-\frac{1}{2}+\sigma+j\right] \prod_{i=0}^{|n|-1} \prod_{j=1}^{i}\left[-\Lambda-\frac{1}{2}-\sigma-j\right] \\
& \times \frac{\prod_{\varepsilon= \pm} G_{q}\left(1-\theta_{*}-\frac{1}{2}+\varepsilon(\sigma+n)\right) \prod_{\varepsilon, \varepsilon^{\prime}= \pm} G_{q}\left(1+\varepsilon(\sigma+n)-\theta_{t}+\varepsilon^{\prime} \theta_{0}\right)}{G_{q}(1+2(\sigma+n)) G_{q}(1-2(\sigma+n))} .
\end{aligned}
$$

Then we have as $\Lambda \rightarrow \infty$ by the definition of $q$-number

$$
\begin{aligned}
q^{\Lambda n^{2}} & \prod_{i=0}^{|n|-1}\left[-\Lambda-\frac{1}{2}+\frac{|n|}{n} \sigma\right] \prod_{i=0}^{|n|-1} \prod_{j=1}^{i}\left[-\Lambda-\frac{1}{2}+\sigma+j\right] \prod_{i=0}^{|n|-1} \prod_{j=1}^{i}\left[-\Lambda-\frac{1}{2}-\sigma-j\right] \\
& \rightarrow(q-1)^{-n^{2}} \prod_{i=0}^{|n|-1} q^{-1 / 2+|n| \sigma / n} \prod_{i=0}^{|n|-1} \prod_{j=1}^{i} q^{-1 / 2+\sigma+j} \prod_{i=0}^{|n|-1} \prod_{j=1}^{i} q^{-1 / 2-\sigma-j} \\
& =(q-1)^{-n^{2}} q^{-n^{2} / 2+\sigma n},
\end{aligned}
$$

and by the identity (1.1) of $q$-Gamma function

$$
\prod_{\varepsilon= \pm}\left(\frac{\Gamma_{q}\left(-\Lambda-\frac{1}{2}+\varepsilon \sigma\right)}{\Gamma_{q}\left(-\Lambda+\frac{1}{2}+\varepsilon \sigma\right)}\right)^{\varepsilon n}=\left(\frac{\left[-\Lambda-\frac{1}{2}-\sigma\right]}{\left[-\Lambda-\frac{1}{2}+\sigma\right]}\right)^{n} \rightarrow q^{-2 \sigma n} .
$$

Therefore we obtain

$$
\begin{aligned}
C_{1} s^{n} C & {\left[\begin{array}{cc}
\theta_{1} & \theta_{t} \\
\theta_{\infty}+\frac{1}{2} & \theta_{0}
\end{array} \mid \sigma+n\right] t^{(\sigma+n)^{2}-\theta_{t}^{2}-\theta_{0}^{2}} } \\
& \rightarrow \tilde{s}^{n}\left(t_{1} / \sqrt{q}\right)^{(\sigma+n)^{2}-\theta_{t}^{2}-\theta_{0}^{2}} C_{\mathrm{V}}\left[\theta_{*}-\frac{1}{2}, \theta_{t}, \theta_{0} \mid \sigma+n\right]
\end{aligned}
$$

as $\Lambda \rightarrow \infty$. Similarly, we can compute the coefficients of $Z$ in the other tau functions and obtain the desired results.

In what follows, we abbreviate $\tau_{i}^{\mathrm{V}}\left(\theta_{*}, \theta_{t}, \theta_{0} \mid s, \sigma, t\right)$ to $\tau_{i}$.

Theorem 3.2. The functions

$$
y=q^{-\theta_{*}-1}(q-1)^{1 / 2} t \frac{\tau_{3} \tau_{4}}{\tau_{1} \tau_{2}}, \quad z=-\frac{\tau_{1} \tau_{2}-\tau_{1} \underline{\tau_{2}}}{q^{\theta_{*} / 2+1 / 2} \tau_{1} \underline{\tau_{2}}}
$$

solves the q-Painlevé $V$ equation

$$
\frac{y \bar{y}}{a_{3} a_{4}}=-\frac{\left(\bar{z}-b_{1} t\right)\left(\bar{z}-b_{2} t\right)}{\bar{z}-b_{3}}, \quad \frac{z \bar{z}}{b_{3}}=-\frac{\left(y-a_{1} t\right)\left(y-a_{2} t\right)}{a_{4}\left(y-a_{3}\right)}
$$

with the parameters

$$
\begin{aligned}
& a_{1}=q^{-\theta_{*}-1}, \quad a_{2}=q^{-2 \theta_{t}-\theta_{*}-1}, \quad a_{3}=q^{-1}, \quad a_{4}=q^{-3 \theta_{*} / 2-1 / 2}, \\
& b_{1}=q^{-\theta_{0}-\theta_{t}-\theta_{*} / 2}, \quad b_{2}=q^{\theta_{0}-\theta_{t}-\theta_{*} / 2}, \quad b_{3}=q^{-\theta_{*} / 2-1 / 2} .
\end{aligned}
$$

Proof. By definition we have

$$
C_{1} C_{2}=(q-1)^{1 / 2} C_{3} C_{4} \text {. }
$$

Hence, by $(3.1)$ the solution $(y, z)$ of the $q$-Painlevé VI equation has the following limit

$$
y \rightarrow y_{1}=q^{-\theta_{*}-1}(q-1)^{1 / 2} t_{1} \frac{\tau_{3} \tau_{4}}{\tau_{1} \tau_{2}}, \quad q^{-\Lambda / 2} z \rightarrow z_{1}=-\frac{\tau_{1} \tau_{2}-\tau_{1} \underline{\tau_{2}}}{q^{\theta_{*} / 2+1 / 2} \tau_{1} \underline{\tau_{2}}}, \quad \Lambda \rightarrow \infty .
$$


Substituting (3.1) into the $q$-Painlevé VI equation (2.2), we get

$$
\begin{aligned}
& \frac{y \bar{y}}{q^{-\Lambda-\theta_{*}-2}}=\frac{\left(\bar{z}-q^{-\theta_{0}-\theta_{t}+\left(\Lambda-\theta_{*}\right) / 2} t_{1}\right)\left(\bar{z}-q^{\theta_{0}-\theta_{t}+\left(\Lambda-\theta_{*}\right) / 2} t_{1}\right)}{\left(\bar{z}-q^{\left(\Lambda-\theta_{*}-1\right) / 2}\right)\left(\bar{z}-q^{\left.-\left(\Lambda+\theta_{*}+1\right) / 2\right)}\right)} \\
& \frac{z \bar{z}}{q^{-1}}=-\frac{\left(y-q^{-\theta_{*}-1} t_{1}\right)\left(y-q^{-2 \theta_{t}-\theta_{*}-1} t_{1}\right)}{\left(y-q^{-1}\right)\left(y-q^{-\Lambda-\theta_{*}-1}\right)}
\end{aligned}
$$

Hence, since $y \rightarrow y_{1}, q^{-\Lambda / 2} z \rightarrow z_{1}$ as $\Lambda \rightarrow \infty$, the system (3.4), (3.5) degenerate to the $q$-Painlevé $\mathrm{V}$ equation (3.3) for $y=y_{1}$ and $z=z_{1}$ as $\Lambda \rightarrow \infty$.

Since we also have

$$
C_{5} C_{6}=(q-1)^{1 / 2} C_{7} C_{8}, \quad C_{1} C_{2}=C_{5} C_{6},
$$

we obtain the following conjecture.

Conjecture 3.3. The tau functions $\tau_{i}(i=1, \ldots, 6)$ satisfy the following bilinear equations

$$
\begin{aligned}
& \tau_{1} \tau_{2}-q^{-\theta_{*}}(q-1)^{1 / 2} t \tau_{3} \tau_{4}-\left(1-q^{-\theta_{*}} t\right) \overline{\tau_{1}} \underline{\tau_{2}}=0, \\
& (q-1)^{-1 / 2} \tau_{1} \tau_{2}-\tau_{3} \tau_{4}+\left(1-q^{-\theta_{*}} t\right) q^{2 \theta_{t}} \underline{{ }_{5}} \overline{\tau_{6}}=0, \\
& (q-1)^{-1 / 2} \tau_{1} \tau_{2}-q^{2 \theta_{t}} \tau_{3} \tau_{4}+q^{2 \theta_{t}} \tau_{5} \tau_{6}=0, \\
& \tau_{1} \underline{\tau_{2}}+q^{\theta_{t}-1 / 2}(q-1)^{1 / 2} t \underline{\tau_{5}} \tau_{6}-\tau_{1} \tau_{2}=0, \\
& (q-1)^{-1 / 2} \tau_{1} \underline{\tau_{2}}+q^{\theta_{0}+2 \theta_{t}} \underline{\tau}_{5} \tau_{6}-q^{\theta_{t}} \underline{\tau}_{3} \tau_{4}=0, \\
& (q-1)^{-1 / 2} \tau_{1} \underline{\tau_{2}}+q^{-\theta_{0}+2 \underline{\theta_{t}}} \tau_{5} \tau_{6}-q^{\theta_{t}} \tau_{3} \underline{\tau_{4}}=0 .
\end{aligned}
$$

Then the functions

$$
y=q^{-\theta_{*}-1}(q-1)^{1 / 2} t \frac{\tau_{3} \tau_{4}}{\tau_{1} \tau_{2}}, \quad z=-q^{\theta_{t}-\theta_{*} / 2-1}(q-1)^{1 / 2} t \frac{\tau_{5} \tau_{6}}{\tau_{1} \underline{\tau_{2}}}
$$

solves $q-\mathrm{P}_{\mathrm{V}}(3.3)$.

The four-term bilinear equation (2.13) admits the following limit.

Proposition 3.4. We have

$$
\underline{\tau_{1}} \tau_{2}-\tau_{1} \underline{\tau_{2}}=\frac{q^{-1 / 2}(q-1)^{1 / 2}}{q^{\theta_{0}}-q^{-\theta_{0}}} t\left(\underline{\tau_{3}} \tau_{4}-\tau_{3} \underline{\tau_{4}}\right) .
$$

Proof. The identity (3.12) is a direct consequence of (2.13) by the limit (3.1) as $\Lambda \rightarrow \infty$.

We remark that tau functions without the Chern-Simons term is also obtained by the limit

$$
\theta_{1}+\theta_{\infty}=-\Lambda, \quad \theta_{1}-\theta_{\infty}=\theta_{*}, \quad s=\tilde{s}(q-1)^{-2 \sigma} \prod_{\varepsilon= \pm} \Gamma_{q}\left(\frac{1}{2}+\Lambda+\varepsilon \sigma\right)^{-\varepsilon}, \quad \Lambda \rightarrow \infty
$$

from the tau functions of $q-\mathrm{P}_{\mathrm{VI}}$. 


\section{From $q-\mathrm{P}_{\mathrm{V}}$ to $q-\mathrm{P}_{\mathrm{III}_{1}}$}

In this section, we take a limit of the tau functions of $q-\mathrm{P}_{\mathrm{V}}$ to $q-\mathrm{P}_{\mathrm{III}_{1}}$. Define the tau function by

$$
\tau^{\mathrm{III}_{1}}\left(\theta_{*}, \theta_{\star} \mid s, \sigma, t\right)=\sum_{n \in \mathbb{Z}} s^{n} t^{(\sigma+n)^{2}} C_{\mathrm{III}_{1}}\left[\theta_{*}, \theta_{\star} \mid \sigma+n\right] Z_{\mathrm{III}_{1}}\left[\theta_{*}, \theta_{\star} \mid \sigma+n, t\right],
$$

with

$$
\begin{aligned}
& C_{\mathrm{III}}\left[\theta_{*}, \theta_{\star} \mid \sigma\right]=(q-1)^{-2 \sigma^{2}} \prod_{\varepsilon= \pm} \frac{G_{q}\left(1-\theta_{*}+\varepsilon \sigma\right) G_{q}\left(1+\varepsilon \sigma-\theta_{\star}\right)}{G_{q}(1+2 \varepsilon \sigma)}, \\
& Z_{\mathrm{III}_{1}}\left[\theta_{*}, \theta_{\star} \mid \sigma, t\right]=\sum_{\left(\lambda_{+}, \lambda_{-}\right) \in \mathbb{Y}^{2}} t^{\left|\lambda_{+}\right|+\left|\lambda_{-}\right|} \frac{\prod_{\varepsilon= \pm} N_{\varnothing, \lambda_{\varepsilon}}\left(q^{-\theta_{*}-\varepsilon \sigma}\right) N_{\lambda_{\varepsilon}, \varnothing}\left(q^{\varepsilon \sigma-\theta_{\star}}\right)}{\prod_{\varepsilon, \varepsilon^{\prime}= \pm} N_{\lambda_{\varepsilon}, \lambda_{\varepsilon^{\prime}}}\left(q^{\left(\varepsilon-\varepsilon^{\prime}\right) \sigma}\right)} .
\end{aligned}
$$

Let us define the tau functions for $q-\mathrm{P}_{\mathrm{III}_{1}}$ by

$$
\begin{aligned}
\tau_{1}^{\mathrm{III}}=\tau^{\mathrm{III}_{1}}\left(\theta_{*}-\frac{1}{2}, \theta_{\star} \mid s, \sigma, t / \sqrt{q}\right), & \tau_{2}^{\mathrm{III}_{1}}=\tau^{\mathrm{III}_{1}}\left(\theta_{*}+\frac{1}{2}, \theta_{\star} \mid s, \sigma, \sqrt{q} t\right), \\
\tau_{3}^{\mathrm{III}_{1}}=\tau^{\mathrm{III}_{1}}\left(\theta_{*}, \theta_{\star}-\frac{1}{2} \mid s, \sigma+\frac{1}{2}, t / \sqrt{q}\right), & \tau_{4}^{\mathrm{III}_{1}}=\tau^{\mathrm{III}_{1}}\left(\theta_{*}, \theta_{\star}+\frac{1}{2} \mid s, \sigma-\frac{1}{2}, \sqrt{q} t\right) .
\end{aligned}
$$

Put

$$
\begin{aligned}
& C_{1}=(q-1)^{-\sigma^{2}} q^{-\Lambda \sigma^{2}-\left(\theta_{t}^{2}+\theta_{0}^{2}\right) / 2} t^{\theta_{t}^{2}+\theta_{0}^{2}} \prod_{\varepsilon= \pm} G_{q}(1-\Lambda+\varepsilon \sigma)^{-1}, \\
& C_{2}=(q-1)^{-\sigma^{2}} q^{-\Lambda \sigma^{2}+\left(\theta_{t}^{2}+\theta_{0}^{2}\right) / 2} t^{\theta_{t}^{2}+\theta_{0}^{2}} \prod_{\varepsilon= \pm} G_{q}(1-\Lambda+\varepsilon \sigma)^{-1}, \\
& C_{3}=(q-1)^{-(\sigma+1 / 2)^{2}} q^{-(\Lambda+1 / 2)(\sigma+1 / 2)^{2}} t^{\theta_{t}^{2}+\left(\theta_{0}+1 / 2\right)^{2}} \prod_{\varepsilon= \pm} G_{q}\left(\frac{1}{2}-\Lambda+\varepsilon\left(\sigma+\frac{1}{2}\right)\right)^{-1}, \\
& C_{4}=(q-1)^{-(\sigma-1 / 2)^{2}} q^{-(\Lambda-1 / 2)(\sigma-1 / 2)^{2}} t^{\theta_{t}^{2}+\left(\theta_{0}-1 / 2\right)^{2}} \prod_{\varepsilon= \pm} G_{q}\left(\frac{3}{2}-\Lambda+\varepsilon\left(\sigma-\frac{1}{2}\right)\right)^{-1}, \\
& C_{5}=(q-1)^{-(\sigma+1 / 2)^{2}} q^{-(\Lambda-1 / 2)(\sigma+1 / 2)^{2}} t^{\left(\theta_{t}-1 / 2\right)^{2}+\theta_{0}^{2}} \prod_{\varepsilon= \pm} G_{q}\left(\frac{3}{2}-\Lambda+\varepsilon\left(\sigma+\frac{1}{2}\right)\right)^{-1}, \\
& C_{6}=(q-1)^{-(\sigma-1 / 2)^{2}} q^{-(\Lambda+1 / 2)(\sigma-1 / 2)^{2}} t^{\left(\theta_{t}+1 / 2\right)^{2}+\theta_{0}^{2}} \prod_{\varepsilon= \pm} G_{q}\left(\frac{1}{2}-\Lambda+\varepsilon\left(\sigma-\frac{1}{2}\right)\right)^{-1} .
\end{aligned}
$$

Proposition 4.1. Set

$$
\begin{aligned}
& \theta_{t}+\theta_{0}=\Lambda, \quad \theta_{t}-\theta_{0}=\theta_{\star}, \quad t=q^{\Lambda} t_{1}, \\
& s=\tilde{s}(q-1)^{-2 \sigma} q^{-\sigma(2 \Lambda+1)} \prod_{\varepsilon= \pm} \Gamma_{q}(-\Lambda+\varepsilon \sigma)^{-\varepsilon} .
\end{aligned}
$$

Then we have

$$
\begin{array}{ll}
C_{i} \tau_{i}^{\mathrm{V}}\left(\theta_{*}, \theta_{t}, \theta_{0} \mid s, \sigma, t\right) \rightarrow \tau_{i}^{\mathrm{III}_{1}}\left(\theta_{*}, \theta_{\star} \mid \tilde{s}, \sigma, t_{1}\right), & i=1,2,3,4, \\
C_{i} \tau_{i}^{\mathrm{V}}\left(\theta_{*}, \theta_{t}, \theta_{0} \mid s, \sigma, t\right) \rightarrow \tau_{i-2}^{\mathrm{III}_{1}}\left(\theta_{*}, \theta_{\star} \mid \tilde{s}, \sigma, q t_{1}\right), & i=5,6,
\end{array}
$$

as $\Lambda \rightarrow \infty$.

Proof. For any partition $\lambda$ we have

$$
N_{\lambda, \varnothing}\left(q^{-\Lambda} u\right) q^{\Lambda|\lambda|}=\prod_{\square \in \lambda}\left(q^{\Lambda}-q^{-\ell_{\lambda}(\square)-a_{\varnothing}(\square)-1} u\right) \rightarrow f_{\lambda}(u)^{-1}, \quad \Lambda \rightarrow \infty .
$$


Hence, the series $Z_{\mathrm{V}}\left[\theta_{*}, \theta_{t}, \theta_{0} \mid \sigma, t\right]$ goes to $Z_{\mathrm{III}_{1}}\left[\theta_{*}, \theta_{\star} \mid \sigma, t_{1}\right]$ as $\Lambda \rightarrow \infty$. The coefficients of $Z_{\mathrm{V}}$ are computed in the same way as in the proof of Proposition 3.1 using (3.2) and we obtain the desired results.

In what follows, we abbreviate $\tau_{i}^{\mathrm{III}_{1}}\left(\theta_{*}, \theta_{\star} \mid s, \sigma, t\right)$ to $\tau_{i}$. Fortunately, the four-term bilinear equation (3.12) degenerates to a three-term bilinear equation.

Proposition 4.2. We have

$$
\underline{\tau_{1}} \tau_{2}-\tau_{1} \underline{\tau_{2}}=q^{-1 / 4} t^{1 / 2} \tau_{3} \underline{\tau_{4}} .
$$

Proof. By definition and (4.1) we have

$$
\begin{aligned}
\underline{C_{1}} C_{2}=C_{1} \underline{C_{2}} & =\left(q^{-\Lambda}-q^{\sigma}\right)(q-1)^{-1 / 2} t_{1}^{-1 / 2} q^{\theta_{0}+1 / 4} \underline{C_{3}} C_{4} \\
& =\left(q^{-\Lambda}-q^{\sigma}\right)(q-1)^{-1 / 2} t_{1}^{-1 / 2} q^{-\theta_{0}+1 / 4} C_{3} \underline{C_{4}} .
\end{aligned}
$$

Hence from the four-term bilinear equation (3.12) degenerates to the three-term bilinear equation (4.2) by (4.1) as $\Lambda \rightarrow \infty$.

Theorem 4.3. The functions

$$
y=q^{-\theta_{*}-1} t^{1 / 2} \frac{\tau_{3} \tau_{4}}{\tau_{1} \tau_{2}}, \quad z=q^{-\theta_{*} / 2-3 / 4} t^{1 / 2} \frac{\tau_{3} \underline{\tau_{4}}}{\underline{\tau_{1}}}
$$

solves the q-Painlevé $\mathrm{III}_{1}$ equation

$$
\frac{y \bar{y}}{a_{3} a_{4}}=-\frac{\bar{z}\left(\bar{z}-b_{2} t\right)}{\bar{z}-b_{3}}, \quad \frac{z \bar{z}}{b_{3}}=-\frac{y\left(y-a_{2} t\right)}{a_{4}\left(y-a_{3}\right)}
$$

with the parameters

$$
a_{2}=q^{-\theta_{\star}-\theta_{*}-1}, \quad a_{3}=q^{-1}, \quad a_{4}=q^{-3 \theta_{*} / 2-1 / 2}, \quad b_{2}=q^{-\theta_{*} / 2}, \quad b_{3}=q^{-\theta_{*} / 2-1 / 2} .
$$

Furthermore, the tau functions $\tau_{i}(i=1, \ldots, 4)$ satisfy the following bilinear equations.

$$
\begin{aligned}
& \tau_{1} \tau_{2}-q^{-\theta_{*}} t^{1 / 2} \tau_{3} \tau_{4}-\overline{\tau_{1}} \underline{\tau_{2}}=0, \\
& \tau_{1} \tau_{2}-q^{\theta \star} t^{-1 / 2} \tau_{3} \tau_{4}+q^{\theta \star} t^{-1 / 2} \overline{\tau_{3}} \underline{\tau_{4}}=0, \\
& \tau_{1} \underline{\tau_{2}}+q^{-1 / 4} t^{1 / 2} \tau_{3} \underline{\tau_{4}}-\underline{\tau_{1}} \tau_{2}=0, \\
& \tau_{1} \underline{\tau_{2}}+q^{1 / 4} t^{-1 / 2} \tau_{3} \underline{\tau_{4}}-q^{1 / 4} t^{-1 / 2} \underline{\tau_{3}} \tau_{4}=0 .
\end{aligned}
$$

Proof. By definition and (4.1) we have

$$
C_{1} C_{2}=\left(q^{-\Lambda}-q^{\sigma}\right)(q-1)^{-1 / 2} t_{1}^{-1 / 2} C_{3} C_{4}
$$

Hence, by (4.1) and (4.2) the solution $(y, z)$ of the $q$-Painlevé V equation degenerates to

$$
y \rightarrow y_{1}=q^{-\theta_{*}-1} t_{1}^{1 / 2} \frac{\tau_{3} \tau_{4}}{\tau_{1} \tau_{2}}, \quad z \rightarrow z_{1}=q^{-\theta_{*} / 2-3 / 4} t_{1}^{1 / 2} \frac{\tau_{3} \underline{\tau_{4}}}{\underline{\tau_{1}}}, \quad \Lambda \rightarrow \infty .
$$

Also, the $q$-Painlevé V equation (3.3) degenerates to the $q$-Painlevé $\mathrm{III}_{1}$ equation (4.4) for $y=y_{1}$ and $z=z_{1}$ as $\Lambda \rightarrow \infty$. 
Next we prove the bilinear equations (4.5)-(4.8). The bilinear equation (4.7) is (4.2). The identity (4.8) is obtained by substituting the expression $(4.3)$ of $(y, z)$ into the $q$-Painlevé III $_{1}$ equation

$$
\frac{y \bar{y}}{a_{3} a_{4}}=-\frac{\bar{z}\left(\bar{z}-b_{2} t\right)}{\bar{z}-b_{3}},
$$

and using the bilinear equation (4.7).

In order to prove (4.5) and (4.6), we use the following transformation

$$
\left(\tilde{\theta}_{*}, \tilde{\theta}_{\star}, \tilde{\sigma}, \tilde{s}, \tilde{t}\right)=\left(-\theta_{\star},-\theta_{*}, \sigma-\frac{1}{2}, C s, q^{-\theta_{*}-\theta_{\star}+1 / 2} t\right),
$$

where

$$
C=q^{(\sigma-1)\left(2 \theta_{*}+2 \theta_{\star}+1\right)} \prod_{\varepsilon, \varepsilon^{\prime}= \pm} \Gamma_{q}\left(\frac{1}{2}+\varepsilon \theta_{*}+\varepsilon^{\prime}(\sigma-1)\right)^{-\varepsilon \varepsilon^{\prime}} \Gamma_{q}\left(\frac{1}{2}+\varepsilon\left(\theta_{\star}+\frac{1}{2}\right)+\varepsilon^{\prime}(\sigma-1)\right)^{-\varepsilon \varepsilon^{\prime}} .
$$

From the definition of the Nekrasov factor, for a partition $\lambda$ we have

$$
N_{\varnothing, \lambda}(u) N_{\lambda, \varnothing}(w)=(u w)^{|\lambda|} N_{\varnothing, \lambda}\left(w^{-1}\right) N_{\lambda, \varnothing}\left(u^{-1}\right) .
$$

By the identity above, the series part $Z$ of the tau functions $\tau_{1}, \ldots, \tau_{4}$ transform to

$$
\begin{aligned}
& Z_{\mathrm{III}_{1}}\left[\tilde{\theta}_{*}-\frac{1}{2}, \tilde{\theta}_{\star} \mid \tilde{\sigma}, \tilde{t} / \sqrt{q}\right]=Z_{\mathrm{III}_{1}}\left[\theta_{*}, \theta_{\star}+\frac{1}{2} \mid \sigma-\frac{1}{2}, \sqrt{q} t\right], \\
& Z_{\mathrm{III}_{1}}\left[\tilde{\theta}_{*}+\frac{1}{2}, \tilde{\theta}_{\star} \mid \tilde{\sigma}, \sqrt{q} \tilde{t}\right]=Z_{\mathrm{III}_{1}}\left[\theta_{*}, \theta_{\star}-\frac{1}{2} \mid \sigma-\frac{1}{2}, \sqrt{q} t\right], \\
& Z_{\mathrm{III}_{1}}\left[\tilde{\theta}_{*}, \tilde{\theta}_{\star}-\frac{1}{2} \mid \tilde{\sigma}+\frac{1}{2}, \tilde{t} / \sqrt{q}\right]=Z_{\mathrm{III}_{1}}\left[\theta_{*}+\frac{1}{2}, \theta_{\star} \mid \sigma, \sqrt{q} t\right], \\
& Z_{\mathrm{III}_{1}}\left[\tilde{\theta}_{*}, \tilde{\theta}_{\star}+\frac{1}{2} \mid \tilde{\sigma}-\frac{1}{2}, \tilde{t}\right]=Z_{\mathrm{III}_{1}}\left[\theta_{*}-\frac{1}{2}, \theta_{\star} \mid \sigma-1, \sqrt{q} t\right],
\end{aligned}
$$

respectively. Using the identity

$$
\frac{G_{q}(1+x+n) G_{q}(1-x)}{G_{q}(1-x-n) G_{q}(1+x)}=(-1)^{n(n+1) / 2} q^{n(n+1) x / 2+(n-1) n(n+1) / 6} \Gamma_{q}(x)^{n} \Gamma_{q}(1-x)^{n}
$$

for $n \in \mathbb{Z}$, we can compute the coefficients $C_{\mathrm{III}_{1}}$ and obtain

$$
\begin{array}{ll}
\tilde{\tau}_{1}=K\left[\theta_{*}, \theta_{\star}+\frac{1}{2}, \sigma-\frac{1}{2}\right] \tau_{4}, & \tilde{\tau}_{2}=s K\left[\theta_{*}, \theta_{\star}-\frac{1}{2}, \sigma-\frac{1}{2}\right] \overline{\tau_{3}}, \\
\tilde{\tau}_{3}=K\left[\theta_{*}+\frac{1}{2}, \theta_{\star}, \sigma\right] \tau_{2}, & \tilde{\tau}_{4}=s K\left[\theta_{*}-\frac{1}{2}, \theta_{\star}, \sigma-1\right] \overline{\tau_{1}},
\end{array}
$$

where we denote by $\tilde{\tau}_{i}$ the tau functions with parameters $\left(\tilde{\theta}_{*}, \tilde{\theta}_{\star}, \tilde{\sigma}, \tilde{s}, \tilde{t}\right)$ and by $\tau_{i}$ the tau functions with parameters $\left(\theta_{*}, \theta_{\star}, \sigma, s, t\right)$, and

$$
K\left[\theta_{*}, \theta_{\star}, \sigma\right]=q^{-\left(\theta_{*}+\theta_{\star}\right) \sigma^{2}} \prod_{\varepsilon, \varepsilon^{\prime}= \pm} G_{q}\left(1+\varepsilon \theta_{*}+\varepsilon^{\prime} \sigma\right)^{\varepsilon} G_{q}\left(1+\varepsilon \theta_{\star}+\varepsilon^{\prime} \sigma\right)^{\varepsilon} .
$$

By definition we have

$$
\frac{K\left[\theta_{*}, \theta_{\star}+\frac{1}{2}, \sigma-\frac{1}{2}\right] K\left[\theta_{*}, \theta_{\star}-\frac{1}{2}, \sigma-\frac{1}{2}\right]}{K\left[\theta_{*}+\frac{1}{2}, \theta_{\star}, \sigma\right] K\left[\theta_{*}-\frac{1}{2}, \theta_{\star}, \sigma-1\right]}=-q^{\left(\theta_{\star}-\theta_{*}\right) / 2} .
$$

Applying the transformation (4.9) to the bilinear equations (4.7) and (4.8) and using the relation (4.10), we obtain the identities (4.5) and (4.6).

We note that the bilinear equations (3.6), (3.8), (3.9), and (3.10) for the tau functions of $q-\mathrm{P}_{\mathrm{V}}$ degenerate to $(4.5),(4.6),(4.7)$, and (4.8), respectively. 


\section{From $q-\mathrm{P}_{\mathrm{III}_{1}}$ to $q-\mathrm{P}_{\mathrm{III}_{2}}$}

In this section, we take a limit of the tau functions of $q-\mathrm{P}_{\mathrm{III}}$ to $q-\mathrm{P}_{\mathrm{III}_{2}}$. Define the tau function by

$$
\tau^{\mathrm{III}_{2}}\left(\theta_{*} \mid s, \sigma, t\right)=\sum_{n \in \mathbb{Z}} s^{n} t^{(\sigma+n)^{2}} C_{\mathrm{III}_{2}}\left[\theta_{*} \mid \sigma+n\right] Z_{\mathrm{III}_{2}}\left[\theta_{*} \mid \sigma+n, t\right],
$$

with

$$
\begin{aligned}
& C_{\mathrm{III}_{2}}\left[\theta_{*} \mid \sigma\right]=(q-1)^{-3 \sigma^{2}} \prod_{\varepsilon= \pm} \frac{G_{q}\left(1-\theta_{*}+\varepsilon \sigma\right)}{G_{q}(1+2 \varepsilon \sigma)} \\
& Z_{\mathrm{III}_{2}}\left[\theta_{*} \mid \sigma, t\right]=\sum_{\left(\lambda_{+}, \lambda_{-}\right) \in \mathbb{Y}^{2}} t^{\left|\lambda_{+}\right|+\left|\lambda_{-}\right|} \frac{\prod_{\varepsilon= \pm} N_{\varnothing, \lambda_{\varepsilon}}\left(q^{-\theta_{*}-\varepsilon \sigma}\right) f_{\lambda_{\varepsilon}}\left(q^{\varepsilon \sigma}\right)^{-1}}{\prod_{\varepsilon, \varepsilon^{\prime}= \pm} N_{\lambda_{\varepsilon}, \lambda_{\varepsilon^{\prime}}}\left(q^{\left(\varepsilon-\varepsilon^{\prime}\right) \sigma}\right)} .
\end{aligned}
$$

In the same way as in Section 3, it is possible to remove $f_{\lambda_{\varepsilon}}\left(q^{\varepsilon \sigma}\right)^{-1}$ from $Z_{\mathrm{III}_{2}}\left[\theta_{*} \mid \sigma, t\right]$ by change of variables. Because if we set

$$
Z_{\mathrm{III}_{2}}^{C S=0}\left[\theta_{*} \mid \sigma, t\right]=\sum_{\left(\lambda_{+}, \lambda_{-}\right) \in \mathbb{Y}^{2}} t^{\left|\lambda_{+}\right|+\left|\lambda_{-}\right|} \frac{\prod_{\varepsilon= \pm} N_{\varnothing, \lambda_{\varepsilon}}\left(q^{-\theta_{*}-\varepsilon \sigma}\right)}{\prod_{\varepsilon, \varepsilon^{\prime}= \pm} N_{\lambda_{\varepsilon}, \lambda_{\varepsilon^{\prime}}}\left(q^{\left(\varepsilon-\varepsilon^{\prime}\right) \sigma}\right)},
$$

then we have

$$
Z_{\mathrm{III}_{2}}\left[\theta_{*} \mid \sigma, t\right]=Z_{\mathrm{III}_{2}}^{C S=0}\left[-\theta_{*} \mid \sigma, q^{-\theta_{*}} t\right] .
$$

Let us define the tau functions for $q-\mathrm{P}_{\mathrm{III}_{2}}$ by

$$
\begin{aligned}
\tau_{1}^{\mathrm{III}}=\tau^{\mathrm{II}_{2}}\left(\theta_{*}-\frac{1}{2} \mid s, \sigma, t / \sqrt{q}\right), & \tau_{2}^{\mathrm{III}_{2}}=\tau^{\mathrm{III}_{2}}\left(\theta_{*}+\frac{1}{2} \mid s, \sigma+1, \sqrt{q} t\right), \\
\tau_{3}^{\mathrm{III}_{2}} & =\tau^{\mathrm{III}_{2}}\left(\theta_{*} \mid s, \sigma+\frac{1}{2}, t\right) .
\end{aligned}
$$

Put

$$
\begin{aligned}
& C_{1}=(q-1)^{-\sigma^{2}} q^{-\Lambda \sigma^{2}} \prod_{\varepsilon= \pm} G_{q}(1-\Lambda+\varepsilon \sigma)^{-1} \\
& C_{2}=C_{1}, \\
& C_{3}=(q-1)^{-(\sigma+1 / 2)^{2}} q^{-(\Lambda-1 / 2)(\sigma+1 / 2)^{2}} \prod_{\varepsilon= \pm} G_{q}\left(\frac{3}{2}-\Lambda+\varepsilon\left(\sigma+\frac{1}{2}\right)\right)^{-1}, \\
& C_{4}=(q-1)^{-(\sigma-1 / 2)^{2}} q^{-(\Lambda+1 / 2)(\sigma-1 / 2)^{2}} \prod_{\varepsilon= \pm} G_{q}\left(\frac{1}{2}-\Lambda+\varepsilon\left(\sigma-\frac{1}{2}\right)\right)^{-1} .
\end{aligned}
$$

Proposition 5.1. Set

$$
\theta_{\star}=\Lambda, \quad t=q^{\Lambda} t_{1}, \quad s=\tilde{s}(q-1)^{-2 \sigma} q^{-\sigma(2 \Lambda+1)} \prod_{\varepsilon= \pm} \Gamma_{q}(-\Lambda+\varepsilon \sigma)^{-\varepsilon} .
$$

Then we have

$$
\begin{aligned}
& C_{i} \tau_{i}^{\mathrm{III} I_{1}}\left(\theta_{*}, \theta_{\star} \mid s, \sigma, t\right) \rightarrow \tau_{i}^{\mathrm{III}_{2}}\left(\theta_{*} \mid \tilde{s}, \sigma, t_{1}\right), \quad i=1,3, \\
& C_{2} \tau_{2}^{\mathrm{III}_{1}}\left(\theta_{*}, \theta_{\star} \mid s, \sigma, t\right) \rightarrow \tilde{s} \tau_{2}^{\mathrm{III}}\left(\theta_{*} \mid \tilde{s}, \sigma, t_{1}\right), \\
& C_{4} \tau_{4}^{\mathrm{III}_{1}}\left(\theta_{*}, \theta_{\star} \mid s, \sigma, t\right) \rightarrow \tilde{s} \tau_{3}^{\mathrm{III} I_{2}}\left(\theta_{*} \mid \tilde{s}, \sigma, t_{1}\right)
\end{aligned}
$$

as $\Lambda \rightarrow \infty$. 
In what follows, we abbreviate $\tau_{i}^{\mathrm{III}_{2}}\left(\theta_{*} \mid s, \sigma, t\right)$ to $\tau_{i}$. Since we have the relation

$$
C_{1} C_{2}=(q-1)^{-1 / 2}\left(q^{-\Lambda / 2}-q^{\Lambda / 2-\sigma}\right) C_{3} C_{4},
$$

we obtain the following theorem by the degeneration.

Theorem 5.2. The functions

$$
y=q^{-\theta_{*}-1}(q-1)^{-1 / 2} t^{1 / 2} \frac{\tau_{3}^{2}}{\tau_{1} \tau_{2}}, \quad z=q^{-\theta_{*} / 2-3 / 4}(q-1)^{-1 / 2} t^{1 / 2} \frac{\tau_{3} \underline{\tau_{3}}}{\underline{\tau_{1}}}
$$

solves the q-Painlevé $\mathrm{III}_{2}$ equation

$$
\frac{y \bar{y}}{a_{3} a_{4}}=-\frac{\bar{z}^{2}}{\bar{z}-b_{3}}, \quad \frac{z \bar{z}}{b_{3}}=-\frac{y\left(y-a_{2} t\right)}{a_{4}\left(y-a_{3}\right)}
$$

with the parameters

$$
a_{2}=q^{-\theta_{*}-1}, \quad a_{3}=q^{-1}, \quad a_{4}=q^{-3 \theta_{*} / 2-1 / 2}, \quad b_{2}=q^{-\theta_{*} / 2}, \quad b_{3}=q^{-\theta_{*} / 2-1 / 2} .
$$

Furthermore, the tau functions $\tau_{i}(i=1,2,3)$ satisfy the following bilinear equations.

$$
\begin{aligned}
& \tau_{1} \tau_{2}-q^{-\theta_{*}}(q-1)^{-1 / 2} t^{1 / 2} \tau_{3}^{2}-\overline{\tau_{1}} \underline{\tau_{2}}=0, \\
& \tau_{1} \tau_{2}-(q-1)^{-1 / 2} t^{-1 / 2} \tau_{3}^{2}+(q-1)^{-1 / 2} t^{-1 / 2} \overline{\tau_{3}} \underline{\tau_{3}}=0, \\
& \tau_{1} \underline{\tau_{2}}+q^{-1 / 4}(q-1)^{-1 / 2} t^{1 / 2} \tau_{3} \underline{\tau_{3}}-\underline{\tau_{1}} \tau_{2}=0 .
\end{aligned}
$$

We note that the bilinear equations (4.5), (4.6), and (4.7) for the tau functions of $q-\mathrm{P}_{\mathrm{III}_{1}}$ degenerate to (5.1), (5.2), and (5.3), respectively.

\section{From $q-\mathrm{P}_{\mathrm{III}_{2}}$ to $q-\mathrm{P}_{\mathrm{III}_{3}}$}

In this section, we take a limit of the tau functions of $q-\mathrm{P}_{\mathrm{III}_{2}}$ to $q-\mathrm{P}_{\mathrm{III}_{3}}$. Define the tau function by

$$
\tau^{\mathrm{III}}(s, \sigma, t)=\sum_{n \in \mathbb{Z}} s^{n} t^{(\sigma+n)^{2}} C_{\mathrm{III}_{3}}[\sigma+n] Z_{\mathrm{III}_{3}}[\sigma+n, t],
$$

with

$$
\begin{aligned}
& C_{\mathrm{III}_{3}}[\sigma]=(q-1)^{-4 \sigma^{2}} \prod_{\varepsilon= \pm} \frac{1}{G_{q}(1+2 \varepsilon(\sigma+n))} \\
& Z_{\mathrm{III}_{3}}[\sigma, t]=\sum_{\left(\lambda_{+}, \lambda_{-}\right) \in \mathbb{Y}^{2}} t^{\left|\lambda_{+}\right|+\left|\lambda_{-}\right|} \frac{1}{\prod_{\varepsilon, \varepsilon^{\prime}= \pm} N_{\lambda_{\varepsilon}, \lambda_{\varepsilon^{\prime}}}\left(q^{\left(\varepsilon-\varepsilon^{\prime}\right) \sigma}\right)} .
\end{aligned}
$$

Let us define the tau functions for $q-\mathrm{P}_{\mathrm{III}_{3}}$ by

$$
\tau_{1}^{\mathrm{III}_{3}}=\tau^{\mathrm{III}_{3}}(s, \sigma, t), \quad \tau_{2}^{\mathrm{III}_{3}}=\tau^{\mathrm{III}_{3}}\left(s, \sigma+\frac{1}{2}, t\right) .
$$

Put

$$
\begin{aligned}
& C_{1}=(q-1)^{-\sigma^{2}} q^{-(\Lambda-1 / 2) \sigma^{2}} \prod_{\varepsilon= \pm} G_{q}\left(\frac{3}{2}-\Lambda+\varepsilon \sigma\right)^{-1} \\
& C_{2}=(q-1)^{-(\sigma+1)^{2}} q^{-(\Lambda+1 / 2)(\sigma+1)^{2}} \prod_{\varepsilon= \pm} G_{q}\left(\frac{1}{2}-\Lambda+\varepsilon(\sigma+1)\right)^{-1} \\
& C_{3}=(q-1)^{-(\sigma+1 / 2)^{2}} q^{-\Lambda(\sigma+1 / 2)^{2}} \prod_{\varepsilon= \pm} G_{q}\left(1-\Lambda+\varepsilon\left(\sigma+\frac{1}{2}\right)\right)^{-1}
\end{aligned}
$$


Proposition 6.1. Set

$$
\theta_{*}=\Lambda, \quad t=q^{\Lambda} t_{1}, \quad s=\tilde{s}(q-1)^{-2 \sigma} q^{-2 \sigma \Lambda} \prod_{\varepsilon= \pm} \Gamma_{q}\left(\frac{1}{2}-\Lambda+\varepsilon \sigma\right)^{-\varepsilon} .
$$

Then we have

$$
\begin{aligned}
& C_{1} \tau_{1}^{\mathrm{III}_{2}}\left(\theta_{*} \mid s, \sigma, t\right) \rightarrow \tau_{1}^{\mathrm{III}_{3}}\left(\tilde{s}, \sigma, t_{1}\right), \\
& C_{2} \tau_{2}^{\mathrm{III}_{2}}\left(\theta_{*} \mid s, \sigma, t\right) \rightarrow \tau_{1}^{\mathrm{III}_{3}}\left(\tilde{s}, \sigma, t_{1}\right) / \tilde{s}, \\
& C_{3} \tau_{3}^{\mathrm{III}_{2}}\left(\theta_{*} \mid s, \sigma, t\right) \rightarrow \tau_{2}^{\mathrm{III}_{3}}\left(\tilde{s}, \sigma, t_{1}\right),
\end{aligned}
$$

as $\Lambda \rightarrow \infty$.

In what follows, we abbreviate $\tau_{i}^{\mathrm{III}_{3}}(s, \sigma, t)$ to $\tau_{i}$. Since we have the relation

$$
C_{1} C_{2}=(q-1)^{1 / 2} \frac{q^{-\sigma-1 / 2+\Lambda / 2}}{q^{-\sigma-1 / 2}-q^{\Lambda}} C_{3}^{2},
$$

we obtain the following theorem by the degeneration.

Theorem 6.2. The functions

$$
y=t^{1 / 2} \frac{s \tau_{2}^{2}}{\tau_{1}^{2}}, \quad z=q^{-3 / 4} t^{1 / 2} \frac{s \tau_{2} \underline{\tau_{2}}}{\tau_{1} \underline{\tau_{1}}}
$$

solves the q-Painlevé $\mathrm{III}_{3}$ equation

$$
\frac{y \bar{y}}{a_{3}}=\bar{z}^{2}, \quad z \bar{z}=-\frac{y\left(y-a_{2} t\right)}{y-a_{3}}
$$

with the parameters

$$
a_{2}=q^{-1}, \quad a_{3}=q^{-1} .
$$

Furthermore, the tau functions $\tau_{1}, \tau_{2}$ satisfy the following bilinear equations.

$$
\begin{aligned}
& s t^{1 / 2} \tau_{2}^{2}-\tau_{1}^{2}+\overline{\tau_{1}} \underline{\tau_{1}}=0, \\
& s^{-1} t^{1 / 2} \tau_{1}^{2}-\tau_{2}^{2}+\overline{\tau_{2}} \underline{\tau_{2}}=0 .
\end{aligned}
$$

We note that the bilinear equations (5.1), (5.2) for the tau functions of $q-\mathrm{P}_{\mathrm{III}_{2}}$ degenerate to (6.2), (6.3), respectively. As suggested in [5, equations (2.9)-(2.11)], the bilinear equation (6.3) is derived from (6.2) by the transformation $\sigma \rightarrow \sigma+1 / 2$.

Remark 6.3. The tau function $\mathcal{T}_{c}\left(q^{2 \sigma}, s ; q \mid t\right)$ proposed in [5] for the $q$-Painlevé $\mathrm{III}_{3}$ equation are related to our tau functions by

$$
\mathcal{T}_{c}\left(q^{2 \sigma}, s ; q \mid t\right)=(-1)^{-2 \sigma^{2}} \tau^{\mathrm{III}}\left((-1)^{-4 \sigma} s, \sigma, t\right) .
$$

Remark 6.4. $q-P\left(A_{7}^{\prime}\right)$ in [19] (or $q-P\left(A_{1}^{(1)} / A_{7}^{(1)}\right)$ in [17, equation (8.14)]) is

$$
\frac{y \bar{y}}{a_{4}}=-\frac{\bar{z}\left(\bar{z}-b_{2} t\right)}{\bar{z}-b_{3}}, \quad \frac{z \bar{z}}{b_{3}}=\frac{y^{2}}{a_{4}},
$$

where $y=y(t), z=z(t)$, and $a_{4}, b_{1}, b_{2}, b_{3}$ are complex parameters. Replacing $y, z$ in (6.1) by $z, \underline{y}$, we obtain $q-P\left(A_{7}^{\prime}\right)$ with $a_{4}=1, b_{2}=1$, and $b_{3}=q^{-1}$. 
The bilinear equations (6.2), (6.3) are also proved by using the Nakajima-Yoshioka blow-up equations [6]. There exists another $q$-difference equation admitting $\mathrm{P}_{\mathrm{III}_{3}}$ and $\mathrm{P}_{\mathrm{I}}$ as limits [13], which corresponds to the $q$-difference Painlevé equation of the surface type $A_{7}^{(1)}$ [25]. Its standard form (see equation (2.44) in [26]) is

$$
\bar{g} g^{2} \underline{g}=t^{2}(1-g),
$$

where $g=g(t)$. A series expansion of the tau function for $q-P\left(A_{7}^{(1)}\right)(6.4)$ was proposed and conjectured to satisfy its bilinear form in [4]. Later, it was proved in [6]. Below, we show that their tau function for $q-P\left(A_{7}^{(1)}\right)(6.4)$ is also obtained as another limit of the tau function for $q-\mathrm{P}_{\mathrm{III}_{2}}$.

Redefine the tau function by

$$
\tau^{\mathrm{III}_{3}}(s, \sigma, t)=\sum_{n \in \mathbb{Z}} s^{n} t^{(\sigma+n)^{2}} C_{\mathrm{III}_{3}}[\sigma+n] Z_{\mathrm{III}_{3}}[\sigma+n, t],
$$

with

$$
\begin{aligned}
& C_{\mathrm{III}_{3}}[\sigma]=(-1)^{n^{2}}(q-1)^{-4 \sigma^{2}} \prod_{\varepsilon= \pm} \frac{1}{G_{q}(1+2 \varepsilon(\sigma+n))}, \\
& Z_{\mathrm{III}_{3}}[\sigma, t]=\sum_{\left(\lambda_{+}, \lambda_{-}\right) \in \mathbb{Y}^{2}} t^{\left|\lambda_{+}\right|+\left|\lambda_{-}\right|} \frac{\prod_{\varepsilon= \pm} f_{\lambda_{\varepsilon}}\left(q^{\varepsilon \sigma}\right)^{-1}}{\prod_{\varepsilon, \varepsilon^{\prime}= \pm} N_{\lambda_{\varepsilon}, \lambda_{\varepsilon^{\prime}}}\left(q^{\left(\varepsilon-\varepsilon^{\prime}\right) \sigma}\right)} .
\end{aligned}
$$

Let us define the tau functions for $q-P\left(A_{7}^{(1)}\right)$ by

$$
\tau_{1}^{\mathrm{III}_{3}}=\tau^{\mathrm{III}_{3}}(s, \sigma, t / \sqrt{q}), \quad \tau_{2}^{\mathrm{III}_{3}}=\tau^{\mathrm{III}_{3}}\left(s, \sigma+\frac{1}{2}, t\right) .
$$

Put

$$
\begin{aligned}
& C_{1}=(q-1)^{-\sigma^{2}} \prod_{\varepsilon= \pm} G_{q}\left(\frac{3}{2}+\Lambda+\varepsilon \sigma\right)^{-1}, \\
& C_{2}=(q-1)^{-(\sigma+1)^{2}} \prod_{\varepsilon= \pm} G_{q}\left(\frac{1}{2}+\Lambda+\varepsilon(\sigma+1)\right)^{-1}, \\
& C_{3}=(q-1)^{-(\sigma+1 / 2)^{2}} \prod_{\varepsilon= \pm} G_{q}\left(1+\Lambda+\varepsilon\left(\sigma+\frac{1}{2}\right)\right)^{-1} .
\end{aligned}
$$

Proposition 6.5. Set

$$
\theta_{*}=-\Lambda, \quad s=\tilde{s}(q-1)^{-2 \sigma} \prod_{\varepsilon= \pm} \Gamma_{q}\left(\frac{1}{2}+\Lambda+\varepsilon \sigma\right)^{-\varepsilon} .
$$

Then we have

$$
\begin{aligned}
& C_{1} \tau_{1}^{\mathrm{III}_{2}}\left(\theta_{*} \mid s, \sigma, t\right) \rightarrow \tau_{1}^{\mathrm{III}_{3}}(\tilde{s}, \sigma, t), \\
& C_{2} \tau_{2}^{\mathrm{III}_{2}}\left(\theta_{*} \mid s, \sigma, t\right) \rightarrow \tau_{1}^{\mathrm{III}_{3}}(\tilde{s}, \sigma, q t) / \tilde{s}, \\
& C_{3} \tau_{3}^{\mathrm{III}_{2}}\left(\theta_{*} \mid s, \sigma, t\right) \rightarrow \tau_{2}^{\mathrm{III}_{3}}(\tilde{s}, \sigma, t),
\end{aligned}
$$

as $\Lambda \rightarrow \infty$.

In what follows, we abbreviate $\tau_{i}^{\mathrm{III}_{3}}(s, \sigma, t)$ to $\tau_{i}$. Since we have the relation

$$
C_{1} C_{2}=\frac{(q-1)^{1 / 2}}{1-q^{\Lambda-\sigma+1 / 2}} C_{3}^{2},
$$

we obtain the following theorem by the degeneration. 
Theorem 6.6. The functions

$$
y=-q^{-1} t^{1 / 2} \frac{s \tau_{2}^{2}}{\tau_{1} \overline{\tau_{1}}}, \quad z=-q^{-3 / 4} t^{1 / 2} \frac{s \tau_{2} \underline{\tau_{2}}}{\underline{\tau_{1}}}
$$

solves

$$
y \bar{y}=-q^{-3 / 2} \frac{\bar{z}^{2}}{\bar{z}-q^{-1 / 2}}, \quad z \bar{z}=y(q y-t) .
$$

Furthermore, the tau functions $\tau_{1}, \tau_{2}$ satisfy the following bilinear equations.

$$
\begin{aligned}
& s^{-1} t^{1 / 2} \tau_{1} \overline{\tau_{1}}-\tau_{2}^{2}+\overline{\tau_{2}} \underline{\tau_{2}}=0, \\
& \tau_{1}^{2}-s q^{-1 / 4} t^{1 / 2} \tau_{2} \underline{\tau_{2}}-\underline{\tau_{1}} \overline{\tau_{1}}=0 .
\end{aligned}
$$

We note that the bilinear equations (5.2), (5.3) for the tau functions of $q-\mathrm{P}_{\mathrm{III}_{2}}$ degenerate to (6.6), (6.7), respectively. By the change of variables $t \rightarrow \sqrt{q} t, \sigma \rightarrow \sigma+1 / 2$, the bilinear equation (6.7) transforms (6.6). The bilinear equation (6.6) is equivalent to the bilinear equa-

tion (4.20) for $N=2, m=1$ in [6], which is for $q-P\left(A_{7}^{(1)}\right)$. Following [4, Example 3.5], we take a time evolution $T$ as $T(f(\sigma, t))=f(\sigma+1 / 2, \sqrt{q} t)$. Then the bilinear equation (6.7) is equivalent to

$$
\tau^{2}-t^{1 / 2} \bar{\tau} \underline{\tau}-\overline{\bar{\tau}} \underline{\underline{\tau}}=0
$$

where $\tau=\tau^{\mathrm{III}_{3}}(s, \sigma, t), \bar{\tau}=T(\tau), \underline{\tau}=T^{-1}(\tau)$. Let $g=t^{1 / 2} \bar{\tau} \underline{\tau} \tau^{-2}$, then $g$ satisfies $q$ $P\left(A_{7}^{(1)}\right)(6.4)$.

\section{Acknowledgements}

This work is partially supported by JSPS KAKENHI Grant Number JP15K17560. The authors thank the referees for valuable suggestions and comments.

\section{References}

[1] Alday L.F., Gaiotto D., Tachikawa Y., Liouville correlation functions from four-dimensional gauge theories, Lett. Math. Phys. 91 (2010), 167-197, arXiv:0906.3219.

[2] Awata H., Feigin B., Shiraishi J., Quantum algebraic approach to refined topological vertex, J. High Energy Phys. 2012 (2012), no. 3, 041, 35 pages, arXiv:1112.6074.

[3] Awata H., Yamada Y., Five-dimensional AGT conjecture and the deformed Virasoro algebra, J. High Energy Phys. 2010 (2010), no. 1, 125, 11 pages, arXiv:0910.4431.

[4] Bershtein M.A., Gavrylenko P.G., Marshakov A.V., Cluster Toda lattices and Nekrasov functions, Theoret. and Math. Phys. 198 (2019), 157-188, arXiv:1804.10145.

[5] Bershtein M.A., Shchechkin A.I., $q$-deformed Painlevé $\tau$ function and $q$-deformed conformal blocks, J. Phys. A: Math. Theor. 50 (2017), 085202, 22 pages, arXiv:1608.02566.

[6] Bershtein M.A., Shchechkin A.I., Painlevé equations from Nakajima-Yoshioka blow-up relations, Lett. Math. Phys., to appear, arXiv:1811.04050.

[7] Bonelli G., Grassi A., Tanzini A., Quantum curves and q-deformed Painlevé equations, Lett. Math. Phys. 109 (2019), 1961-2001, arXiv:1710.11603.

[8] Bonelli G., Lisovyy O., Maruyoshi K., Sciarappa A., Tanzini A., On Painlevé/gauge theory correspondence, Lett. Math. Phys. 107 (2017), 2359-2413, arXiv:1612.06235.

[9] Felder G., Müller-Lennert M., Analyticity of Nekrasov partition functions, Comm. Math. Phys. 364 (2018), 683-718, arXiv:1709.05232. 
[10] Gamayun O., Iorgov N., Lisovyy O., Conformal field theory of Painlevé VI, J. High Energy Phys. 2012 (2012), no. 10, 038, 25 pages, arXiv:1207.0787.

[11] Gamayun O., Iorgov N., Lisovyy O., How instanton combinatorics solves Painlevé VI, V and IIIs, J. Phys. A: Math. Theor. 46 (2013), 335203, 29 pages, arXiv:1302.1832.

[12] Gambier B., Sur les équations différentielles du second ordre et du premier degré dont l'intégrale générale est a points critiques fixes, Acta Math. 33 (1910), 1-55.

[13] Grammaticos B., Ramani A., Parameterless discrete Painlevé equations and their Miura relations, J. Nonlinear Math. Phys. 23 (2016), 141-149.

[14] Iorgov N., Lisovyy O., Teschner J., Isomonodromic tau-functions from Liouville conformal blocks, Comm. Math. Phys. 336 (2015), 671-694, arXiv:1401.6104.

[15] Jimbo M., Nagoya H., Sakai H., CFT approach to the $q$-Painlevé VI equation, J. Integrable Syst. 2 (2017), xyx009, 27 pages, arXiv:1706.01940.

[16] Jimbo M., Sakai H., A q-analog of the sixth Painlevé equation, Lett. Math. Phys. 38 (1996), 145-154.

[17] Kajiwara K., Noumi M., Yamada Y., Geometric aspects of Painlevé equations, J. Phys. A: Math. Theor. 50 (2017), 073001, 164 pages, arXiv:1509.08186.

[18] Mano T., Asymptotic behaviour around a boundary point of the $q$-Painlevé VI equation and its connection problem, Nonlinearity 23 (2010), 1585-1608.

[19] Murata M., Lax forms of the q-Painlevé equations, J. Phys. A: Math. Theor. 42 (2009), 115201, 17 pages, arXiv:0810.0058.

[20] Nagoya H., Irregular conformal blocks, with an application to the fifth and fourth Painlevé equations, J. Math. Phys. 56 (2015), 123505, 24 pages, arXiv:1505.02398.

[21] Nagoya H., Remarks on irregular conformal blocks and Painlevé III and II tau functions, in Proceedings of the Meeting for Study of Number Theory, Hopf Algebras and Related Topics, Yokohama Publ., Yokohama, 2019, 105-124, arXiv:1804.04782.

[22] Painlevé P., Mémoire sur les équations différentielles dont l'intégrale générale est uniforme, Bull. Soc. Math. France 28 (1900), 201-261.

[23] Painlevé P., Sur les équations différentielles du second ordre et d'ordre supérieur dont l'intégrale générale est uniforme, Acta Math. 25 (1902), 1-85.

[24] Ramani A., Grammaticos B., Hietarinta J., Discrete versions of the Painlevé equations, Phys. Rev. Lett. 67 (1991), 1829-1832.

[25] Sakai H., Rational surfaces associated with affine root systems and geometry of the Painlevé equations, Comm. Math. Phys. 220 (2001), 165-229.

[26] Sakai H., Problem: discrete Painlevé equations and their Lax forms, in Algebraic, Analytic and Geometric Aspects of Complex Differential Equations and their Deformations. Painlevé Hierarchies, RIMS Kôkyûroku Bessatsu, Vol. B2, Res. Inst. Math. Sci. (RIMS), Kyoto, 2007, 195-208.

[27] Tachikawa Y., Five-dimensional Chern-Simons terms and Nekrasov's instanton counting, J. High Energy Phys. 2004 (2004), no. 2, 050, 13 pages, arXiv:hep-th/0401184.

[28] Yanagida S., Norm of the Whittaker vector of the deformed Virasoro algebra, arXiv:1411.0462. 